Homology, Homotopy and Applications, vol.20(2), 2018, pp.239-257

\title{
RACKS AS MULTIPLICATIVE GRAPHS
}

\author{
JACOB MOSTOVOY
}

\author{
(communicated by Ronald Brown)
}

\begin{abstract}
We interpret augmented racks as a certain kind of multiplicative graphs and show that this point of view is natural for defining rack homology. We also define the analogue of the group algebra for these objects; in particular, we see how discrete racks give rise to Hopf algebras and Lie algebras in the Loday-Pirashvili category $\mathcal{L} \mathcal{M}$. Finally, we discuss the integration of Lie algebras in $\mathcal{L} \mathcal{M}$ in the context of multiplicative graphs and augmented racks.
\end{abstract}

\section{Introduction}

Racks are self-distributive algebraic structures which arise in different contexts, such as knot theory or the structure theory of Hopf algebras. They have been invented several times under different names: wracks (which later morphed into racks), distributive groupoids, automorphic sets. A union of conjugacy classes in a group can be considered as a rack; there are many other interesting examples. A closely related algebraic structure is that of an augmented rack or a crossed $G$-set: this is a rack together with a morphism into a group (which is thought of as a rack with the operation of conjugation). We refer to [5] for an overview of the subject; we shall use the facts from that paper without explicit reference.

In the present note we interpret augmented racks as multiplicative, rather than self-distributive structures; we call these structures group-like graphs. This point of view provides a simple interpretation of rack spaces (and, hence, rack homology) and leads to the definition of an analogue of the group algebra for racks: it is a Hopf algebra in the Loday-Pirashvili category of linear maps. This algebra carries a filtration similar to the filtration by the powers of the augmentation ideal in a group algebra; the associated graded Hopf algebra is the universal enveloping algebra of a certain Lie algebra in the Loday-Pirashvili category, which can be described in terms of what we call the graded coinvariant module of the corresponding augmented rack. Note that Hopf dialgebras and Leibniz algebras are particular cases of Hopf algebras and Lie algebras in the Loday-Pirashvili category, see [10], so our constructions can be translated into the dialgebra language.

We also consider smooth group-like graphs and indicate how to set up a version of Lie theory for them. One can think of a Lie algebra in the Loday-Pirashvili category

Received October 7, 2017, revised February 14, 2018; published on June 13, 2018.

2010 Mathematics Subject Classification: 20N99, 05C99, 16 T05.

Key words and phrases: rack, multiplicative graph, Loday-Pirashvili category, Leibniz algebra.

Article available at http://dx.doi.org/10.4310/HHA.2018.v20.n2.a12

Copyright (C) 2018, Jacob Mostovoy. Permission to copy for private use granted. 
(in particular, a Leibniz algebra) as of an infinitesimal object corresponding to a group-like graph of a certain kind, namely, a linear Lie graph. Every such finitedimensional Lie algebra can be integrated to a linear Lie graph; this is consistent with the formal integration procedure of [14]. Linear Lie graphs are in one-to-one correspondence with linear augmented Lie racks; the exponential map of a Lie algebra to the corresponding Lie group is an example of such rack. On the level of nonaugmented racks, this integration procedure has been discussed in [9], where the connection between Leibniz algebras and racks was made for the first time.

Much of what we do is valid not only for group-like graphs, but for multiplicative graphs: if group-like graphs are thought of as being analogous to groups, the multiplicative graphs are analogous to semigroups (and are different from shelves of [2]). We give a concrete example of a multiplicative graph which is not group-like; it arises in knot theory when considering knots with double points. A by-product of our constructions is a functor that assigns a differential graded Lie algebra to a Lie algebra in the Loday-Pirashvili category (in particular, to a Leibniz algebra).

\section{Multiplicative graphs and augmented racks}

\subsection{Multiplicative graphs}

In this note, unless stated otherwise, by a "graph" we shall mean a directed graph, possibly with loops and multiple edges. Such a graph $Q$ can be written as a pair of sets (vertices $V$ and arrows $A$ ) with a pair of maps from arrows to vertices (source $s$ and target $t)$ :

$$
Q=(V \underset{t}{\stackrel{s}{\leftrightarrows}} A)
$$

Recall that the Cartesian product $Q_{1} \square Q_{2}$ of two graphs $Q_{1}=\left(V_{1} \leftleftarrows A_{1}\right)$ and $Q_{2}=$ $\left(V_{2} \leftleftarrows A_{2}\right)$ is a graph on the set of vertices $V_{1} \times V_{2}$, the set of arrows

$$
A_{1} \times V_{2} \sqcup V_{1} \times A_{2}
$$

and the source and target maps

$$
s=s_{1} \times \mathrm{id} \sqcup \mathrm{id} \times s_{2}
$$

and

$$
t=t_{1} \times \mathrm{id} \sqcup \mathrm{id} \times t_{2} .
$$

If $Q_{1}$ and $Q_{2}$ are thought of as 1-dimensional cell complexes with directed 1-cells, the graph $Q_{1} \square Q_{2}$ is obtained from the 2-dimensional cell complex $Q_{1} \times Q_{2}$ by erasing the 2-cells. The Cartesian product of graphs is associative in the sense that there is a canonical isomorphism

$$
\left(Q_{1} \square Q_{2}\right) \square Q_{3} \simeq Q_{1} \square\left(Q_{2} \square Q_{3}\right) .
$$

Definition 2.1. A Cartesian-multiplicative or, simply, multiplicative graph is a graph $Q$ together with a morphism

$$
\mu: Q \square Q \rightarrow Q,
$$

which is associative in the sense that

$$
\mu \circ(\mu \square \mathrm{id})=\mu \circ(\mathrm{id} \square \mu) .
$$


The set of vertices of a multiplicative graph $Q$ is a semigroup. Call $Q$ group-like if this semigroup is, actually, a group.

As an algebraic structure, a group-like graph is equivalent to what is known as an augmented rack. Recall that an augmented rack $X$ over a group $G$ (also called a crossed $G$-set) is a $G$-set $X$ together with a morphism (augmentation map) of $G$-sets $\pi: X \rightarrow G$, where $G$ acts on itself by conjugation. Probably, the most basic example of an augmented rack is a union of an arbitrary set of conjugacy classes in a group $G$.

A group-like graph $Q=(G \leftleftarrows A)$ gives rise to an augmented rack in the following fashion. The "multiplication" $\mu$ gives a two-sided action of the group $G$ of vertices of $Q$ on the set $A$ of arrows. Let $X \subset A$ be the set of arrows whose source is 1 . Then there is an action of $G$ on $X$ defined by

$$
x^{g}=g^{-1} \cdot x \cdot g
$$

and the target map $t: X \rightarrow G$ is a morphism of $G$-actions where $G$ acts on itself by conjugation. In other words, $X$ is an augmented rack over $G$.

Conversely, given an augmented rack $\pi: X \rightarrow G$ one constructs a group-like graph

$$
(G \leftleftarrows G \times X)
$$

by setting

$$
s(g, x)=g, \quad t(g, x)=g \pi(x),
$$

and

$$
\mu\left(\left(g_{1}, x_{1}\right), g_{2}\right)=\left(g_{1} g_{2}, x_{1}^{g_{2}}\right), \quad \mu\left(g_{1},\left(g_{2}, x_{2}\right)\right)=\left(g_{1} g_{2}, x_{2}\right) .
$$

Group-like graphs form a category, whose morphisms are the morphisms of graphs that respect the multiplication; in particular, they are group homomorphisms on vertices. Augmented racks also form a category whose morphisms are the commutative squares of the form

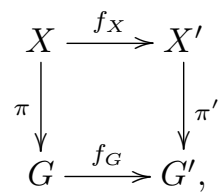

where $f_{G}$ is a homomorphism and $f_{X}(x)^{f_{G}(g)}=f_{X}\left(x^{g}\right)$.

The preceding construction that allows to pass from a group-like graph to a rack and vice versa is functorial:

Theorem 2.2. The category of group-like graphs is equivalent to the category of augmented racks.

There are variations on the definition of a multiplicative graph. One may also consider usual graphs, that is, undirected graphs without loops and multiple edges. These lead to a very particular class of racks. Namely, in this context, for a group-like graph $Q$ with the group of vertices $G$, the set of edges $X$ one of whose ends is the unit of $G$ is a subset of $G$ which is (a) invariant under conjugation; (b) contains together with each $g \in G$ its inverse. ${ }^{1}$ Conversely, for each union $X$ of non-trivial conjugacy

${ }^{1}$ This means that the rack $X$ has a good involution, see [11]. 
classes in $G$ satisfying (b) there exists a group-like graph whose set of vertices is $G$ and whose set of edges that connect to the unit is $X$.

Another variation consists in considering $k$-graphs ${ }^{2}$ instead of graphs, that is, pairs of sets with $k+1$ maps between them, for any $k \geqslant-1$. One defines the Cartesian product of $k$-graphs in the same fashion. For instance, when $k=0$, with this definition each group-like 0 -graph is of the form $(G \leftarrow G \times X)$ where $X$ is a $G$-set and the map is the projection onto the first factor. A group-like 0-graph can be considered as a group-like 1-graph whose only arrows are loops.

As the referee points out, one is tempted to compare the concept of a group-like graph with that of a category; more precisely, an internal category in the category of groups, see [7]. Both kinds of structures are directed graphs whose vertices form a group; however, the arrows in a group-like graph cannot be composed as morphisms. While an internal category in the category of groups (in other words, a crossed module of groups) is an example of an augmented rack, it is an example of a very special kind. In turn, an augmented rack is a particular example of a crossed module of racks [3].

\subsection{Racks without augmentation and their associated groups}

Historically, augmented racks are secondary objects as compared to racks. A rack (without an augmentation) is a set $X$ with a binary operation $X \times X \rightarrow X$, written as

$$
(x, y) \rightarrow x^{y}
$$

satisfying the identity

$$
\left(x^{y}\right)^{z}=\left(x^{z}\right)^{\left(y^{z}\right)}
$$

and such that for every $y, z \in X$ there exists a unique $x \in X$ with

$$
x^{y}=z \text {. }
$$

This definition axiomatized the properties of conjugation in a group: if $X$ is a union of conjugacy classes in a group, the rack structure on $X$ is given by

$$
x^{y}=y^{-1} x y .
$$

The rack structure on a group $G$ given by conjugation is called the conjugation rack of $G$.

In an augmented rack $\pi: X \rightarrow G$, the set $X$ naturally has the structure of a rack with the operation defined as

$$
x^{y}=x^{\pi(y)} .
$$

Conversely, each rack $X$ defines the associated group $G_{X}$ with the presentation

$$
\left\langle\tau_{x_{1}}, \tau_{x_{2}}, \ldots \mid \tau_{x_{i}} \tau_{x_{j}}=\tau_{x_{j}} \tau_{\left(x_{i}{ }^{x_{j}}\right)}\right\rangle,
$$

where $X=\left\{x_{1}, x_{2}, \ldots\right\}$. The group $G_{X}$ acts on $X$ and the tautological map $\tau: X \rightarrow$ $G_{X}$ which sends $x \in X$ to the corresponding generator $\tau_{x}$ of $G_{X}$ is $G_{X}$-equivariant; that is, to say, defines an augmented rack. The functor $X \rightarrow G_{X}$ is left adjoint to the functor of taking the conjugation rack of a group.

\footnotetext{
${ }^{2}$ This terminology is somewhat arbitrary.
} 
Proposition 2.3. The group-like graph corresponding to the augmented rack $X \rightarrow$ $G_{X}$ is path-connected.

Proof. By construction, $X$ can be identified with the set of edges of the graph that emanate from $1 \in G_{X}$; in particular, each generator $\tau_{x} \in G_{X}$ is connected to 1 by the edge $x$. Also, the inverse of a generator $\tau_{x}^{-1}$ is connected to 1 by the edge $\tau_{x}^{-1} \cdot x$. Now, if $w \in G_{X}$ is connected to 1 by a sequence of edges $a_{1}, \ldots, a_{n}$, then $w \tau_{x}$ is also connected to 1 , by the sequence of edges $a_{1}, \ldots, a_{n}, w \cdot x$, and $w \tau_{x}^{-1}$ - by the sequence $a_{1}, \ldots, a_{n}, w \tau_{x}^{-1} \cdot x$.

Note that the only property of the rack $X \rightarrow G_{X}$ that we used in the above proof is that the image of $X$ in $G_{X}$ generates the whole group $G_{X}$. A similar argument gives the following statement:

Proposition 2.4. For an augmented rack $X \rightarrow G$, let $H$ be the subgroup of $G$ generated by the image of $X$. Then $H$ is normal in $G$; it is the group of vertices of the connected component of the unit in the group-like graph of $X \rightarrow G$.

As we shall see later, the connectivity of a group-like graph is reflected in the properties of the bialgebras associated with it.

\section{The associated spaces}

\subsection{The cubical complexes $E Q$ and $B Q$}

Let $\mathrm{Q}_{1}$ be the graph with two vertices and one arrow connecting them; set

$$
\mathrm{Q}_{n}=\mathrm{Q}_{1} \square \cdots \square \mathrm{Q}_{1}
$$

( $n$ factors). A $k$-face of $\mathrm{Q}_{n}$ is a subgraph of $\mathrm{Q}_{n}$ isomorphic to $\mathrm{Q}_{k}$, obtained by replacing $n-k$ of the copies of $\mathrm{Q}_{1}$ in the product above by one of its vertices.

For a graph $Q$, we shall refer to the morphisms $\mathrm{Q}_{n} \rightarrow Q$ as the $n$-cubes of $Q$. The restriction of an $n$-cube to one of the $k$-faces of $\mathrm{Q}_{n}$ is a $k$-face of the $n$-cube. Each arrow $a$ of $Q$ gives rise to a canonical map $a: \mathrm{Q}_{1} \rightarrow Q$. In a multiplicative $Q$, a product $n$-cube is an $n$-cube of the form

$$
\mathrm{Q}_{n}=\mathrm{Q}_{1} \square \cdots \square \mathrm{Q}_{1} \stackrel{a_{1} \square \cdots \square a_{n}}{\longrightarrow} Q \square \cdots \square Q \stackrel{\mu_{n}}{\longrightarrow} Q,
$$

where $a_{i}$ are arrows of $Q$ and the last map is the multiplication of $n$ factors in $Q$; we shall denote such $n$-cube by $a_{1} \square \cdots \square a_{n}$. A product square is a product 2-cube.

Lemma 3.1. The $k$-faces of a product $n$-cube are product $k$-cubes.

Proof. If $\mathrm{Q}_{0}$ is the graph consisting of one vertex and no arrows, we have $\mathrm{Q}_{0} \square \mathrm{Q}_{1}=$ $\mathrm{Q}_{1}$. An $(n-1)$-face of an $n$-cube is obtained by replacing a copy of $\mathrm{Q}_{1}$ in the product by a $Q_{0}$ which can be grouped with the copy of $Q_{1}$ that precedes or follows it; this establishes the lemma.

For a multiplicative graph $Q=(G \leftleftarrows A)$ let $E Q$ be the cubical complex ${ }^{3}$ whose $n$-dimensional faces correspond to the product $n$-cubes of $Q$ and the face maps - to

${ }^{3}$ By a "cubical complex" we mean a geometric realization of a precubical set. 
the inclusions of the $(n-1)$-faces into $\mathrm{Q}_{n}$. The semigroup $G$ of vertices of $Q$ acts on the product $n$-cubes by

$$
\left(g, a_{1} \square \cdots \square a_{n}\right) \mapsto\left(g \cdot a_{1}\right) \square \cdots \square a_{n},
$$

for $g \in G$ acting on $A$ the left via $\mu$. This action is compatible with the face maps and, hence, descends to an action of $G$ on $E Q$. We shall denote the orbit space of this action by $B Q$.

Given a product $k$-cube $a=a_{1} \square \cdots \square a_{k}$ and a product $m$-cube $b=b_{1} \square \cdots \square b_{m}$, one defines the product $(k+m)$-cube $a \square b$ as $a_{1} \square \ldots \square a_{k} \square b_{1} \square \ldots \square b_{m}$. This operation on product cubes is compatible with the face maps so that there is an associative product

$$
E Q \times E Q \rightarrow E Q
$$

given by $(a, b) \mapsto a \square b$.

For group-like graphs, the product cubes are easy to describe:

Lemma 3.2. Let $Q=(G \leftleftarrows A)$ be a group-like graph which gives rise to a rack $X$. Product $n$-cubes in $Q$ are in one-to-one correspondence with $(n+1)$-tuples of the form $\left(g, x_{1}, \ldots, x_{n}\right)$ with $g \in G$ and $x_{i} \in X$.

Proof. Recall that $A=G \times X$. We shall see that each product $n$-cube can be uniquely written as

$$
\left(g, x_{1}\right) \square\left(1, x_{2}\right) \square \cdots \square\left(1, x_{n}\right),
$$

with $g \in G$ and $x_{i} \in X$.

Let us first consider product squares. The product square $\left(g_{1}, x_{1}\right) \square\left(g_{2}, x_{2}\right)$ is of the form

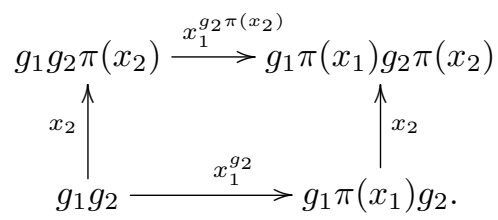

Here the corners of the square are vertices of $Q$, the arrows are labelled by the elements of $X$ rather than $G \times X$ since the corresponding element of $G$ is indicated at the source of the arrow. Now, assume that we are given arbitrary $h=g_{1} g_{2}, z_{1}=x_{1}^{g_{2}}$ and $z_{2}=x_{2}$. Then, the above product square is of the form

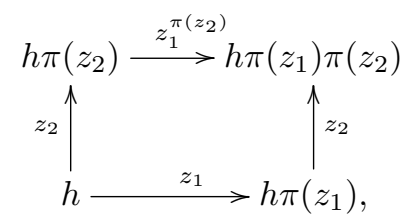

and this establishes the lemma for product squares.

The case of the $n$-cubes with $n>2$ now follows by induction from the associativity of the $\square$ operation. 
In the notation of Lemma 3.2, the action of $G$ on $E Q$ is of the form

$$
\left(g,\left(g^{\prime}, x_{1}, \ldots, x_{n}\right)\right) \mapsto\left(g g^{\prime}, x_{1}, \ldots, x_{n}\right) ;
$$

in particular, it is free and $E Q$ is a covering space of $B Q$.

Lemma 3.3. Let $Q$ be a group-like graph which gives rise to a rack $X$. The space $B Q$ coincides with the rack space of $X$.

The proof is immediate and follows from the definition of the rack space [6] and the description of the product squares given in the proof of Lemma 3.2.

\subsection{The spaces $E Q$ and $B Q$ via the James reduced product}

The cubical complex $E Q$ can be defined in terms of generators and relations. Consider $Q$ as a topological space (one-dimensional simplicial or cubical complex) with a two-sided continuous $G$-action; the group $G$ is then the 0 -skeleton of $Q$. Write $F(Q)$ for the free monoid generated by the points of $Q$ whose identity is the vertex corresponding to $1 \in G$. The monoid $F(Q)$ carries the natural topology induced by the topology on $Q$; it is known as the James reduced product of $Q$, see [8].

Consider the following set of equivalence relations on $F(Q)$ :

$$
g * x \sim g \cdot x, \quad x * g \sim x \cdot g,
$$

for each $g \in G, x \in Q$, where $*$ is the product in $F(Q)$ and $\cdot$ denotes the $G$-action on $Q$.

Proposition 3.4. For a group-like graph $Q$, the monoid $E Q$ is the quotient of $F(Q)$ by the above equivalence relations.

Proof. Denote the congruences (1) by $R$. The space $F(Q) / R$ has the natural structure of a cubical complex. Indeed, $F(Q)$ is the union of cells of the form $q_{1} * \cdots * q_{m}$ where each $q_{i}$ is either a fixed element of $G$ or varies over a fixed arrow in $A$. The congruences $R$ respect this cell subdivision of $F(Q)$; each cell in $F(Q) / R$ can be written as $a_{1} *$ $\cdots * a_{m}$ with $a_{i} \in A$ and $m \geqslant 0$ (in particular, the product may be empty; this gives the unique 0 -cell). Moreover, modulo $R$, each of the cells of positive dimension can be uniquely written as $g * x_{1} * \cdots * x_{n}$ where the $x_{i}$ are edges emanating from 1 and $n \geqslant 1$.

The space $E Q$ contains a copy of $Q$ so that there is a unique continuous surjective homomorphism of $F(Q)$ to $E Q$ which descends to a map

$$
F(Q) / R \rightarrow E Q
$$

that maps $a_{1} * \cdots * a_{n}$ to $a_{1} \square \cdots \square a_{n}$. The preimage of a cell $\left(g, x_{1}, \ldots, x_{n}\right)$ is precisely the cell $g * x_{1} * \cdots * x_{n}$, so that this homomorphism is, actually, an isomorphism.

Remark 3.5. This construction of the rack space makes sense in a somewhat more general context. Let $Y$ be a topological space and $G \subset Y$ a subset with the group structure such that there is a left ${ }^{4}$ action of $G$ on $Y$ extending the left multiplication

\footnotetext{
${ }^{4}$ The same construction works for right and for two-sided actions.
} 
on $G$. Denote by $E(Y, G)$ the quotient of the free monoid $F(Y)$ by the relations

$$
g * x \sim g \cdot x
$$

for all $g \in G, x \in Y$. There is a left action of $G$ on $E(Y, G)$ and we can define $B(Y, G)$ as the quotient space of $E(Y, G)$ by this action. For instance, given a left $G$-set $X$, one can take $Y=G \sqcup X$. A possibly more interesting example is a Cayley graph for $G$ or, indeed, a graph determined by an arbitrary subset $S \subseteq G$ : we set $Y$ to be the graph whose vertices are elements of $G$ and whose edges are pairs $(g, g s)$ for all $g \in G$ and $s \in S$. Note that the 1 -skeleton of $E(Y, G)$ in this case is a multiplicative graph into which the Cayley graph is embedded.

\subsection{The action of $\pi_{1} B Q$ on $\pi_{n} E Q$}

The fundamental group of any topological monoid (or, indeed, of any space with a unital multiplication) is abelian. More generally, let $E$ be a topological monoid and $G \subset E$ be a subgroup acting on $E$ by left translations with the quotient space $B$. Assume that $p: E \rightarrow B$ is a covering. Then, $\pi_{1} E$ lies in the centre of $\pi_{1} B$; in particular, for a group-like graph $Q$ the subgroup $\pi_{1} E Q$ lies in the centre of $\pi_{1} B Q$.

The proof is a standard exercise in topology. Let $p(1)$ be the basepoint in $B$; for a curve $\gamma$ starting at $p(1)$ write $\bar{\gamma}$ for its lifting to $E$ with $\bar{\gamma}(0)=1$. Let $\alpha, \beta:[0,1] \rightarrow B$ be two closed paths starting and ending at $p(1)$ and assume that $\bar{\alpha}$ is closed in $E$. Denote by $\bar{\alpha}_{\tau}$ and $\bar{\beta}_{\tau}$ the reparametrizations of $\bar{\alpha}$ and $\bar{\beta}$, respectively, which are constant outside of the interval $[\tau, \tau+1 / 2]$. Note that the pointwise product curves $\bar{\alpha}_{\tau_{1}} \bar{\beta}_{\tau_{2}}$ are fixed-end homotopic in $E$ for all $\tau_{1}, \tau_{2} \in[0,1 / 2]$; since $\bar{\alpha}$ is closed, their projections to $B$ define closed loops in $B$. Now, writing $\circ$ for the concatenation of loops in $B$ we see that

$$
\overline{\alpha \circ \beta}=\bar{\alpha}_{0} \bar{\beta}_{1 / 2}
$$

while

$$
\overline{\beta \circ \alpha}=\bar{\beta}_{0} \bar{\alpha}_{1 / 2} \sim \bar{\beta}_{1 / 2} \bar{\alpha}_{0}=\bar{\alpha}_{0} \bar{\beta}_{1 / 2},
$$

which means that $\alpha \circ \beta$ equals $\beta \circ \alpha$ as an element of $\pi_{1} B$.

A very similar argument shows that $\pi_{1} B$ acts trivially on $\pi_{n} E$ for all $n$. Since for $n>1$ the groups $\pi_{n} E$ coincide with $\pi_{n} B$, we see that the rack space $B Q$ is always homotopy simple, the fact that was first proved in [6].

\section{Examples}

\subsection{Path graphs}

Probably, the simplest non-trivial example of a multiplicative graph is the graph of pairs of elements in a (semi)group. For a semigroup $G$, let $A=G \times G$ with the maps $s$ and $t$ being the projections onto the first and on the second factors respectively, and the two-sided action of $G$ being the action of the diagonal in $G \times G$ by multiplication. When $G$ is a group, the corresponding augmented rack is the identity map $G \rightarrow G$ (and the non-augmented rack is the conjugation rack of $G$ ).

An extension of this example is the graph whose arrows are sequences of $n$ elements in $G$, with $s$ and $t$ being the first and the last element of the sequence. When $G$ is a topological semigroup, one can consider the graph whose edges are directed paths 
on $G$; again, with $s$ and $t$ being the beginning and the end of the path. If $G$ is a Lie group, it makes sense to consider the group-like graph $L G$ whose arrows are directed segments of geodesics in $G$, parametrized by length. An important feature of this graph is that the maps

$$
s, t: L G \rightarrow G
$$

are vector bundles such that the action of $G$ on $L G$ is linear on the fibres. Indeed, each geodesic $\gamma$ starting at $g \in G$ is determined by a tangent vector in $T_{g} G$ whose direction coincides with that of $\gamma$ and whose length gives the length of $\gamma$, and, therefore, the fibre of $s$ over $g \in G$ can be identified with $T_{g} G$; the same argument goes for the map $t$. This is the most basic example of a linear Lie graph, see Section 6.1.

\subsection{Multiplicative cubical complexes and simplicial monoids}

For any cubical complex with an associative cubical multiplication on it, the 1skeleton is a multiplicative graph. Each multiplicative graph $Q$ can be obtained in such a way, being the 1-skeleton of the cubical complex EQ. Here, as before, a cubical complex is a geometric realization of a precubical set $X$ (a "cubical set without degenerations") and a cubical multiplication is a geometric realization of an associative map $X \times X \rightarrow X$. Recall that the $n$-cubes of the product of two precubical sets $X$ and $Y$ are pairs of cubes of $X$ and $Y$ whose dimensions sum up to $n$.

Also, the 1-skeleton of a simplicial monoid is a multiplicative graph whose vertices are the 0 -simplices, whose edges are the 1 -simplices and whose source and target maps are the face maps. The monoid of 0 -simplices can be identified with the monoid of the degenerate 1-simplices, and, therefore, acts on the 1-simplices by multiplication; since the face maps are homomorphisms, this gives a multiplicative graph. When the monoid of 0-simplices is, actually, a group, its action respects the non-degeneracy of 1simplices. In particular, in this case, one obtains a multiplicative graph whose vertices are the 0 -simplices of the simplicial monoid and whose arrows are the non-degenerate 1-simplices.

\subsection{Knot racks as multiplicative graphs}

One of the most useful examples of racks is the rack associated with a framed knot. In terms of multiplicative graphs, this construction has the following form.

Let $K$ be a parametrized framed knot in $\mathbb{R}^{3}$. Choose a basepoint in the exterior of $K$ and let $A$ be the set of all homotopy classes of smooth loops in $\mathbb{R}^{3}$ which start at the basepoint and cross the knot $K$ exactly once with the positive sign (this means that at the crossing point the tangent vector to the loop, the tangent vector to the knot and the framing vector form a positive basis in $\mathbb{R}^{3}$ ). Each $a \in A$ gives rise to two elements of the knot group $\pi_{1}\left(\mathbb{R}^{3}-K\right)$ as follows. Represent $a$ by a curve $\gamma$ and define $s(a)$ as the class of the loop obtained by moving $\gamma$ at the crossing point off the knot in the direction opposite to the framing; similarly, $t(a)$ is obtained by shifting $\gamma$ off $K$ along the framing. These maps are evidently well-defined. Moreover, there is a twosided action of $\pi_{1}\left(\mathbb{R}^{3}-K\right)$ on $A$ by pre- and post-composing the loops which cross the knot once with the loops that never cross the knot. Therefore $\left(\pi_{1}\left(\mathbb{R}^{3}-K\right) \leftleftarrows A\right)$ is a group-like graph.

It is quite clear that this construction indeed is equivalent to the usual knot rack. Indeed, if $s(a)$ is trivial, a loop $\gamma$ representing $a$ bounds a disk in the exterior of $K$. 
This disk can be squeezed, without moving the crossing point of $\gamma$ with $K$, to an interval connecting the basepoint with a point on the knot; this is how the knot rack is normally defined.

The space $E Q$ for a knot rack also has a natural definition in terms of the curves which cross the knot. Namely, the set of homotopy classes of loops that intersect the knot $n$ times, each of them positively, coincides with the set of product $n$-cubes in the corresponding group-like graph. Indeed, each loop that crosses the knot $n$ times is a concatenation of $n$ loops that hit the knot exactly once. Such a decomposition is not unique; however, the product cube made of these $n$ loops is well-defined.

We should also note that, just as in the case of usual knot racks, here framed knots in $\mathbb{R}^{3}$ can be replaced by $n$-2-dimensional framed submanifolds of an $n$ manifold, or even by $n-k$-dimensional submanifolds with $k>2$. In this latter case, the fundamental group of the knot exterior should be replaced by its $k-1$ st homotopy group. Probably, the most basic example of a graph defined by a codimension two subset is the group-like graph associated with the complement of an $n$-point set in $\mathbb{R}^{2}$. Its vertices are the elements of the free group on $n$ generators $x_{1}, \ldots, x_{n}$ and the arrows are in one-to-one correspondence with pairs of elements of the form $\left(a b, a x_{k} b\right)$ for some $x_{k}$. The rack that corresponds to such a graph is the union of the conjugacy classes of the generators in the free group.

\subsection{String links with double points}

An interesting example of a multiplicative graph which is not group-like is provided by string links with one double point.

Given $n>0$, denote by $L_{n}$ the monoid of isotopy classes of string links on $n$ strands and let $L_{n}^{\bullet}$ be the set of isotopy classes of string links with one transversal double point (see [4] for the definitions). A string link with a double point can be composed with a usual string link on either side and this gives two commuting actions

$$
L_{n} \times L_{n}^{\bullet} \rightarrow L_{n}^{\bullet}
$$

and

$$
L_{n}^{\bullet} \times L_{n} \rightarrow L_{n}^{\bullet}
$$

There are also two maps $L_{n}^{\bullet} \rightarrow L_{n}$ given by the positive and the negative resolution of the double point according to the Vassiliev skein relation; these two maps are compatible with the actions of $L_{n}$ on $L_{n}^{\bullet}$. This means that $\mathcal{L}_{n}:=\left(L_{n} \leftleftarrows L_{n}^{\bullet}\right)$ is a multiplicative graph.

The corresponding space $E \mathcal{L}_{n}$ has been mentioned in the literature; namely, it was constructed by Matveev and Polyak in [13, page 229].

The graph $\mathcal{L}_{n}$ has a group-like subgraph which consists of pure braids with one double point. Two pure braids involved in the resolution of a double point differ by an insertion of a generator: if the positive resolution can be written as $a b$, the negative is of the form $a A_{i j} b$, where $i$ and $j$ are the numbers of the strands which cross at the double point. ${ }^{5}$ The corresponding rack is the union of the conjugacy classes of the generators in the pure braid group.

\footnotetext{
${ }^{5}$ There is a freedom of choosing the sign in the Vassiliev skein relation for string links which involves the relative order in each pair of strands; this is the same as choosing between a generator and its inverse for each $i, j$.
} 


\section{Hopf algebras associated with multiplicative graphs}

Any group $G$ gives rise to two Hopf algebras. One is the group algebra $\mathbf{k}[G]$; it carries the filtration by the powers of the augmentation ideal $I(G)$. The associated graded Hopf algebra $\mathcal{D}(G)$ is the universal enveloping algebra of the graded Lie algebra coming form the lower central series of $G$. In this section we construct the analogs of both Hopf algebras for group-like graphs.

\subsection{The Loday-Pirashvili category}

Let us review some notions from [10]. The Loday-Pirashvili category $\mathcal{L} \mathcal{M}$ over a given field $\mathbf{k}$ (which we assume to be of characteristic zero) has, as objects, pairs of vector spaces

$$
(U \stackrel{f}{\longrightarrow} V)
$$

over $\mathbf{k}$ together with a linear map between them. The morphisms are the commutative squares of the form

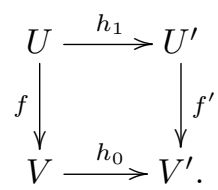

The category $\mathcal{L} \mathcal{M}$ is a tensor category with the tensor product ${ }^{6}$

$$
(U \stackrel{f}{\longrightarrow} V) \otimes\left(U^{\prime} \stackrel{f^{\prime}}{\longrightarrow} V^{\prime}\right)=\left(U \otimes V^{\prime}+V \otimes U^{\prime} \stackrel{f \otimes \mathrm{Id}+\mathrm{Id} \otimes f^{\prime}}{\longrightarrow} V \otimes V^{\prime}\right) .
$$

The natural explanation for this tensor product comes from considering $\mathcal{L} \mathcal{M}$ as the category of 1-jets of differential graded vector spaces, that is, the quotient of the category of (non-negatively graded) chain complexes over $\mathbf{k}$ by the subcategory of the chain complexes with trivial components in degrees 0 and 1.

One may speak of Lie algebras and Hopf algebras in $\mathcal{L M}$; the Milnor-Moore and the Poincaré-Birkhoff-Witt theorems are then valid in $\mathcal{L} \mathcal{M}$. An algebra in $\mathcal{L} \mathcal{M}$ is a pair

$$
(\mathcal{A} \stackrel{f}{\longrightarrow} \mathcal{H})
$$

with $\mathcal{H}$ an algebra, $\mathcal{A}$ an $\mathcal{H}$-bimodule and $f$ a bimodule map. A bialgebra in $\mathcal{L} \mathcal{M}$ has, in addition, a compatible dual structure, namely a coproduct $\Delta_{0}: \mathcal{H} \rightarrow \mathcal{H} \otimes \mathcal{H}$ which makes $\mathcal{H}$ into a bialgebra, and the two-sided coaction

$$
\Delta_{1}: \mathcal{A} \rightarrow \mathcal{A} \otimes \mathcal{H}+\mathcal{H} \otimes \mathcal{A},
$$

which is an $\mathcal{H}$-bimodule map satisfying

$$
\Delta_{0} \circ f=(f \otimes \operatorname{Id}+\operatorname{Id} \otimes f) \circ \Delta_{1} .
$$

A bialgebra is a Hopf algebra if it has an antipode; we shall state the properties satisfied by the antipode in Section 5.3. Hopf algebras in $\mathcal{L} \mathcal{M}$ are related to YetterDrinfel'd modules, see [12].

\footnotetext{
${ }^{6}$ Hereafter we denote by "+" the direct sum of vector spaces.
} 
A Lie algebra in $\mathcal{L} \mathcal{M}$ is a pair

$$
(M \stackrel{f}{\longrightarrow} \mathfrak{g}),
$$

where $\mathfrak{g}$ is a Lie algebra, $M$ is a right ${ }^{7}$ module over $\mathfrak{g}$ and $f$ is a $\mathfrak{g}$-module morphism. In this situation, there exists a bracket on $M$ that gives it the structure of a Leibniz algebra:

$$
[x, y]=[x, f(y)],
$$

where the bracket on the right-hand side denotes the right action of $\mathfrak{g}$ on $M$. Each Leibniz algebra $L$ can be obtained this way by taking the Lie algebra $\mathfrak{g}$ to be the maximal antisymmetric quotient of $L$ and the map $f$ to be the corresponding quotient map. We refer to [10] for more details.

If $M$, considered as a Leibniz algebra, is, actually, a Lie algebra on which $\mathfrak{g}$ acts by derivations, the Lie algebra $(M, \mathfrak{g})$ is a differential crossed module. Differential crossed modules can be identified with the differential graded Lie algebras whose only non-zero terms are in degrees 0 and 1 .

\subsection{Lie algebras in $\mathcal{L} \mathcal{M}$ and differential graded Lie algebras}

There is one important observation that we should make even though it will not be used in what follows: the Lie algebras in $\mathcal{L} \mathcal{M}$ are related to the differential graded Lie algebras in the same way as the multiplicative graphs are related to the products on cubical complexes.

Namely, given a differential graded Lie algebra $\mathfrak{g}_{*}$ :

$$
\cdots \stackrel{d}{\longrightarrow} \mathfrak{g}_{1} \stackrel{d}{\longrightarrow} \mathfrak{g}_{0}
$$

the map $\mathfrak{g}_{1} \stackrel{d}{\longrightarrow} \mathfrak{g}_{0}$ together with the restriction of the bracket of $\mathfrak{g}_{*}$ to the maps $\mathfrak{g}_{1} \otimes \mathfrak{g}_{0} \rightarrow \mathfrak{g}_{1}$ and $\mathfrak{g}_{0} \otimes \mathfrak{g}_{0} \rightarrow \mathfrak{g}_{0}$ is a Lie algebra in $\mathcal{L} \mathcal{M}$.

Conversely, given a Lie algebra $\mathfrak{g}=\left(\mathfrak{g}_{1} \stackrel{d}{\longrightarrow} \mathfrak{g}_{0}\right)$ in $\mathcal{L} \mathcal{M}$ with the Lie bracket denoted by $[\cdot, \cdot]$, consider the free Lie algebra on the vector space $\mathfrak{g}_{0}+\mathfrak{g}_{1}$. It is a differential graded Lie algebra with the differential induced by $d$; we denote its Lie bracket by $\llbracket \cdot, \cdot \rrbracket$. Let $E \mathfrak{g}$ be the quotient of this free differential graded Lie algebra by the relations

$$
\llbracket x, y \rrbracket=[x, y],
$$

where either $x, y \in \mathfrak{g}_{0}$, or $x \in \mathfrak{g}_{1}$ and $y \in \mathfrak{g}_{0}$.

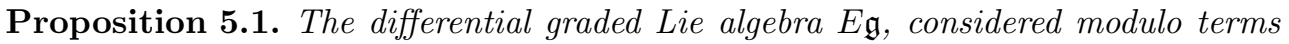
of degree two and higher, coincides with $\mathfrak{g}$ as a Lie algebra in $\mathcal{L} \mathcal{M}$.

The proof is immediate. Observe that the functor $\mathfrak{g} \mapsto E \mathfrak{g}$ from $\mathcal{L} \mathcal{M}$ to DGLA is the left adjoint to the functor of reduction modulo terms of degree 2 and higher.

\footnotetext{
${ }^{7}$ We take right, rather than left, modules so as to have the same conventions as [10].
} 


\subsection{The multiplicative graph algebra}

Let $Q=(G \leftleftarrows A)$ be a multiplicative graph. Write $\mathbf{k}[A]$ for the $\mathbf{k}$-vector space spanned by $A$ and consider the linear map

$$
\phi: \mathbf{k}[A] \rightarrow \mathbf{k}[G]
$$

to the semigroup algebra of $G$ defined by

$$
\phi(a)=t(a)-s(a)
$$

for all $a \in A$. Then $(\mathbf{k}[A] \stackrel{\phi}{\rightarrow} \mathbf{k}[G])$ is an algebra in the Loday-Pirashvili category $\mathcal{L} \mathcal{M}$; the two-sided action of $\mathbf{k}[G]$ on $\mathbf{k}[A]$ is the linear extension of the two-sided action of $G$ on $A$.

Recall that the algebra $\mathbf{k}[G]$ carries a coproduct, which we denote by $\Delta_{0}$ :

$$
\Delta_{0}(g)=g \otimes g
$$

for all $g \in G$. Define the two-sided coaction $\Delta_{1}$ by setting

$$
\Delta_{1}(a)=a \otimes t(a)+s(a) \otimes a,
$$

for all $a \in A$. Note that $\phi$ sends $\Delta_{1}$ to $\Delta_{0}$; moreover, $\Delta_{1}$ is a $\mathbf{k}[G]$-bimodule map. Indeed, for $a \in A$ and $g \in G$ we have

$$
\begin{aligned}
\Delta_{1}(a \cdot g) & =a \cdot g \otimes t(a \cdot g)+s(a \cdot g) \otimes a \cdot g \\
& =a \cdot g \otimes t(a) g+s(a) g \otimes a \cdot g=\Delta_{1}(a) \cdot \Delta_{0}(g) .
\end{aligned}
$$

Similarly, $\Delta_{1}(g \cdot a)=\Delta_{0}(g) \cdot \Delta_{1}(a)$. As a consequence, we have

Lemma 5.2. $(\mathbf{k}[A] \stackrel{\phi}{\rightarrow} \mathbf{k}[G])$ is a bialgebra in $\mathcal{L} \mathcal{M}$.

When $G$ is a group, we can define the involution $S_{1}: \mathbf{k}[A] \rightarrow \mathbf{k}[A]$ as

$$
a \mapsto-s(a)^{-1} \cdot a \cdot t(a)^{-1},
$$

for each $a \in A$. It maps under $\phi$ to the antipode $S_{0}: \mathbf{k}[G] \rightarrow \mathbf{k}[G]$ that sends each group element $g$ to $g^{-1}$. In order to check that $S_{1}$ gives rise to an antipode in $\mathcal{L M}$ we need to verify that for any $a \in A$

$$
\mu \circ(S \otimes \operatorname{Id})_{1} \circ \Delta_{1}=\mu \circ(\operatorname{Id} \otimes S)_{1} \circ \Delta_{1}=0 .
$$

Here $\mu$ is the product, $(S \otimes \mathrm{Id})_{1}$ stands for

and $(\operatorname{Id} \otimes S)_{1}$ for

$$
S_{1} \otimes \operatorname{Id}_{\mathbf{k}[G]}+S_{0} \otimes \operatorname{Id}_{\mathbf{k}[A]}
$$

$$
\operatorname{Id}_{\mathbf{k}[A]} \otimes S_{0}+\operatorname{Id}_{\mathbf{k}[G]} \otimes S_{1}
$$

respectively. Substituting these expressions into (2), we obtain

$$
\left(-s(a)^{-1} \cdot a \cdot t(a)^{-1}\right) t(a)+s(a)^{-1} \cdot a=0
$$

and

$$
a \cdot t(a)^{-1}+s(a) \cdot\left(-s(a)^{-1} \cdot a \cdot t(a)^{-1}\right)=0 .
$$

We have proved

Proposition 5.3. When $Q$ is group-like, $(\mathbf{k}[A] \stackrel{\phi}{\rightarrow} \mathbf{k}[G])$ is a Hopf algebra in $\mathcal{L} \mathcal{M}$.

Note that, in general, this Hopf algebra is not cocommutative. 


\subsection{The augmentation filtration and the associated Hopf algebra}

Write $I(G)$ for the augmentation ideal in $\mathbf{k}[G]$ and let $I^{n}(A) \subset \mathbf{k}[A]$ be the subspace

$$
\left\langle v_{1} \cdot a \cdot v_{2} \mid a \in A, v_{1} \in I^{k}(G), v_{2} \in I^{n-k}(G), k \leqslant n\right\rangle .
$$

The image of $I^{n}(A)$ under $\phi$ lies in $I^{n+1}(G)$.

Lemma 5.4. If $Q$ is group-like and path-connected, $\phi\left(I^{n}(A)\right)=I^{n+1}(G)$.

Proof. It is sufficient to show that $\phi(A)=I(G)$.

The augmentation ideal $I(G)$ is additively generated by the elements of the form $g-1$ with $g \in G$. Since $Q$ is path-connected, for any $g \in G$ there is a path $a_{1}, \ldots, a_{n}$, with $a_{i} \in A$ connecting 1 and $g$, although not necessarily according to the directions of the $a_{i}$. Let $e_{i}=1$ if $a_{i}$ is directed along the path and $e_{i}=-1$ otherwise. Then

$$
\phi\left(\sum e_{i} a_{i}\right)=g-1
$$

which shows that $\phi(A)$ coincides with $I(G)$.

Remark 5.5. For a not necessarily path-connected $Q$, the vertices of the connected component that contains the unit in $G$ form a normal subgroup $G_{0}$. Let $I\left(G, G_{0}\right)$ be the kernel of the homomorphism $\mathbf{k}[G] \rightarrow \mathbf{k}\left[G / G_{0}\right]$ induced by the quotient map. Then $\phi\left(I^{n}(A)\right)$ can be explicitly identified as $I^{n+1}\left(G, G_{0}\right)$.

From now on we shall assume that $Q$ is group-like and path-connected.

The maps $\Delta_{1}$ and $S_{1}$ respect the filtration by the $I^{n}(A)$ so there is a graded Hopf algebra $\left(\mathcal{D}(Q) \stackrel{\phi_{*}}{\longrightarrow} \mathcal{D}(G)\right)$ associated with it. The map $\phi_{*}$, induced by $\phi$, raises the degree by one.

Lemma 5.6. $\left(\mathcal{D}(Q) \stackrel{\phi_{*}}{\longrightarrow} \mathcal{D}(G)\right)$ is an irreducible cocommutative Hopf algebra.

Proof. We have

$$
\Delta_{1}(a)=a \otimes s(a)+s(a) \otimes a+a \otimes \phi(a) .
$$

The "non-cocommutative part" of $\Delta_{1}$

$$
\Delta_{1}^{\prime}: a \mapsto a \otimes \phi(a)
$$

vanishes on the associated graded level since $\phi_{*}$ raises the degree by one. Indeed, for any $u \in \mathbf{k}[A]$ and any $g \in G$ we have

$$
\Delta_{1}^{\prime}(g \cdot u)=(g \otimes g) \cdot \Delta_{1}^{\prime}(u),
$$

and hence,

$$
\Delta_{1}^{\prime}((g-1) \cdot u)=((g-1) \otimes(g-1)+1 \otimes(g-1)+(g-1) \otimes 1) \cdot \Delta_{1}^{\prime}(u) .
$$

The same kind of equality holds for $\Delta_{1}^{\prime}(u \cdot(g-1))$. Moreover, $\Delta_{1}^{\prime}(a) \in A \otimes I(G)$ for any $a \in A$. These formulae show that $\Delta_{1}^{\prime}$ increases the filtration index by one and, therefore, induces the zero map on $\mathcal{D}(Q)$. This implies that the coproduct in the bialgebra $(\mathcal{D}(Q) \rightarrow \mathcal{D}(G))$ is cocommutative. The irreducibility follows from the irreducibility of $\mathcal{D}(G)$. 
Since $\mathcal{D}(G)$ satisfies the conditions of the Milnor-Moore Theorem, $\left(\mathcal{D}(Q) \stackrel{\phi_{*}}{\longrightarrow}\right.$ $\mathcal{D}(G)$ ) also does (see [10]); therefore, it is the universal enveloping algebra of a certain Lie algebra $(M \rightarrow \mathfrak{g})$ in $\mathcal{L} \mathcal{M}$ :

$$
\left(\mathcal{D}(Q) \stackrel{\phi_{*}}{\longrightarrow} \mathcal{D}(G)\right) \simeq(U(\mathfrak{g}) \otimes M \rightarrow U(\mathfrak{g})) .
$$

The Lie algebra $\mathfrak{g}=\operatorname{Prim} \mathcal{D}(G)$ is the graded Lie algebra of the successive quotients of the lower central series of $G$, tensored with $\mathbf{k}$. As for $M$, it can be understood in terms of the graded coinvariant module of the augmented rack corresponding to $Q$.

\subsection{The coinvariant module of an augmented rack}

Let $\pi: X \rightarrow G$ be an augmented rack. The vector space $\mathbf{k}[X]$ spanned by $X$ has a decreasing filtration by the subspaces

$$
I^{n}(X)=\left\langle x^{\left(g_{1}-1\right) \cdots\left(g_{n}-1\right)} \mid x \in X, g_{i} \in G\right\rangle,
$$

where we use the exponential notation for the linear extension of the action of $G$ to an action of $\mathbf{k}[G]$ on $\mathbf{k}[X]$. We should warn that this notation might be not entirely intuitive; for instance, $x^{(g-1)}=x^{g}-x$. However, we want to keep clear the distinction between the $G$-action on $X$ and the two $G$-actions in a group-like graph. When $n=0$, we set $I^{0}(X)=\mathbf{k}[X]$.

Set, for $n>0$

$$
\mathcal{P}^{n}(X):=I^{n}(X) / I^{n+1}(X) ;
$$

in other words, the space $\mathcal{P}^{n}(X)$ consists of the coinvariants of the $G$-action on $I^{n}(X)$. In particular, $\mathcal{P}^{0}(X)=\mathbf{k}[X / G]$ is the vector space spanned by the orbits of the action of $G$ on $X$. Write $\mathcal{P}(X)$ for the graded vector space whose part of degree $k$ is $\mathcal{P}^{k}(X)$.

It is clear from the definition that the space $\mathcal{P}(X)$ is a graded module over $\mathcal{D}(G)$; we call it the coinvariant module. Moreover, the map $\pi$ induces a degree 1 map of graded $\mathcal{D}(G)$-modules $\pi_{*}: \mathcal{P}(X) \rightarrow \mathcal{D}(G)$ :

$$
\left(x^{\left(g_{1}-1\right) \cdots\left(g_{n}-1\right)} \bmod I^{n+1}(X)\right) \mapsto\left((\pi(x)-1)^{\left(g_{1}-1\right) \cdots\left(g_{n}-1\right)} \bmod I^{n+2}(G)\right) .
$$

Indeed, for $u \in I^{k}(G)$ and $g \in G$ we have

$$
u^{g}-u=g^{-1}(u g-g u)=g^{-1}(u(g-1)-(g-1) u) \in I^{k+1}(G)
$$

so that $\pi_{*}$ is well-defined. The image of $\pi_{*}$ in $\mathcal{D}(G)$ lies in the Lie algebra Prim $\mathcal{D}(G)$ of the primitive elements of $\mathcal{D}(G): \pi_{*} \mathcal{P}^{0}(X)$ consists of elements of degree one in $\mathcal{D}(X)$, which are primitive, and $\pi_{*} \mathcal{P}^{n}(X)$ is spanned by the commutators with $\pi_{*} \mathcal{P}^{n-1}(X)$. This shows that $\left(\mathcal{P}(X) \stackrel{\pi_{*}}{\longrightarrow} \operatorname{Prim} \mathcal{D}(G)\right)$ is a graded Lie algebra in $\mathcal{L} \mathcal{M}$.

Proposition 5.7. The Hopf algebra $\left(\mathcal{D}(Q) \stackrel{\phi_{*}}{\longrightarrow} \mathcal{D}(G)\right)$ is the universal enveloping algebra of the Lie algebra $\left(\mathcal{P}(X) \stackrel{\pi_{*}}{\longrightarrow} \operatorname{Prim} \mathcal{D}(G)\right)$, where $X$ is the augmented rack corresponding to the group-like graph $Q$.

Proof. The universal enveloping algebra of $\left(\mathcal{P}(X) \stackrel{\pi_{*}}{\rightarrow} \operatorname{Prim} \mathcal{D}(G)\right)$ is the map

$$
\left(\mathcal{D}(G) \otimes \mathcal{P}(X) \stackrel{\mu\left(\operatorname{Id} \otimes \pi_{*}\right)}{\longrightarrow} \mathcal{D}(G)\right),
$$

where $\mu$ is the product in $\mathcal{D}(G)$, with the following $\mathcal{D}(G)$-bimodule structure on 
$\mathcal{D}(G) \otimes \mathcal{P}(X)$

$$
\begin{gathered}
w_{1} \cdot\left(w_{2} \otimes m\right)=\left(w_{1} w_{2}\right) \otimes m, \\
(w \otimes m) \cdot a=(w a) \otimes m+w \otimes m^{a},
\end{gathered}
$$

for all $w_{1}, w_{2}, w \in \mathcal{D}(G), m \in \mathcal{P}(X)$ and $a \in \operatorname{Prim} \mathcal{D}(G)$ (see [10, page 271]).

Identify the vector space $\mathbf{k}[A]$ with $\mathbf{k}[G] \otimes \mathbf{k}[X]$ via $(g, x) \mapsto g \otimes x$. Under this identification, $I^{n}(A)$ is sent to

$$
\sum_{p+q=n} I^{p}(G) \otimes I^{q}(X)
$$

namely,

$$
\left(g_{1}-1\right) \cdots\left(g_{p}-1\right) \cdot(g, x)^{\left(h_{1}-1\right) \cdots\left(h_{q}-1\right)} \mapsto\left(g_{1}-1\right) \cdots\left(g_{p}-1\right) g \otimes x^{\left(h_{1}-1\right) \cdots\left(h_{q}-1\right)} .
$$

Therefore, as a graded vector space, $\mathcal{D}(Q)$ is isomorphic to $\mathcal{D}(G) \otimes \mathcal{P}(X)$. Under this identification, the map $\phi_{*}$ coincides with $\mu\left(\operatorname{Id} \otimes \pi_{*}\right)$. Also, the $\mathcal{D}(G)$-bimodule structure is the same as that of the universal enveloping algebra of $(\mathcal{P}(X) \rightarrow \operatorname{Prim} \mathcal{D}(G))$. This is clear for the left module structure (3). As for the right module structure, the action on the right by $a \in \mathfrak{g}$ can be represented by a right action of $h-1$ with $h \in G$ :

$$
(u \otimes x)^{h-1}=(u \otimes x)^{h}-u \otimes x=u(h-1) \otimes x^{h}+u \otimes x^{h-1},
$$

with $u \in I^{m}(A)$ and $x \in I^{n}(X)$. It is, actually, sufficient to consider $a \in \operatorname{Prim} \mathcal{D}(G)$ of degree one, since elements of this kind generate $\mathcal{D}(G)$; that is, consider the above equality modulo $I^{m+n+2}$. Then, the right-hand side of the above formula is equivalent to

$$
u(h-1) \otimes x+u \otimes x^{h-1},
$$

which in $\mathcal{D}(Q)$ translates precisely into (4).

\subsection{Edge-like elements in Hopf algebras and the Malcev completion}

Let $(\mathcal{A} \rightarrow \mathcal{H})$ be a Hopf algebra in $\mathcal{L} \mathcal{M}$. Call an element $a \in \mathcal{A}$ edge-like if

$$
\Delta_{1}(a)=a \otimes t(a)+s(a) \otimes a,
$$

where $s(a)$ and $t(a)$ are group-like elements of $\mathcal{H}$. Let us denote the set of group-like elements of $\mathcal{H}$ by $\mathcal{G}_{0}(\mathcal{H})$ and the set of edge-like elements of $\mathcal{A}$ by $\mathcal{G}_{1}(\mathcal{A})$. Assigning to an edge-like element $a$ the corresponding group-like elements $s(a)$ and $t(a)$ we define two maps

$$
s, t: \mathcal{G}_{1}(\mathcal{A}) \rightarrow \mathcal{G}_{0}(\mathcal{H}) .
$$

The two-sided action of $\mathcal{H}$ on $\mathcal{A}$ restricts to the action of the group $\mathcal{G}_{0}(\mathcal{H})$ on $\mathcal{G}_{1}(\mathcal{A})$. We have:

Lemma 5.8. The pair $\left(\mathcal{G}_{0}(\mathcal{H}) \leftleftarrows \mathcal{G}_{1}(\mathcal{A})\right)$ is a group-like graph.

Consider the group-like graph Hopf algebra $\phi: \mathbf{k}[A] \rightarrow \mathbf{k}[G]$. Since $\phi$ maps $I^{n}(A)$ to $I^{n+1}(G)$, it descends to a map

$$
\phi_{n}: \mathbf{k}[A] / I^{n}(A) \rightarrow \mathbf{k}[G] / I^{n+1}(G),
$$

which is also a Hopf algebra in $\mathcal{L} \mathcal{M}$. There is a canonical morphism of $\phi_{n+1}$ onto $\phi_{n}$ 
for each $n$; the inverse limit of the $\phi_{i}$ in $\mathcal{L M}$ is a complete Hopf algebra

$$
\phi^{\wedge}: \mathbf{k}[A]^{\wedge} \rightarrow \mathbf{k}[G]^{\wedge}
$$

whose edge-like elements form a group-like graph

$$
\mathcal{G}_{0}\left(\mathbf{k}[G]^{\wedge}\right) \leftleftarrows \mathcal{G}_{1}\left(\mathbf{k}[A]^{\wedge}\right)
$$

that we call the Malcev completion of $(G \leftleftarrows A)$. Here, a complete Hopf algebra in $\mathcal{L} \mathcal{M}$ is defined as usual: the tensor products in the definition of the comultiplication should be replaced by the completed tensor products. The definition of edge-like elements in a complete Hopf algebra also uses completed tensor products instead of the usual tensor products.

\section{Linear Lie graphs and linear augmented racks}

\subsection{Lie theory}

One can consider multiplicative graphs such that both $G$ and $A$ are smooth manifolds and $s$ and $t$ are submersions onto their image ${ }^{8}$. Our principal motivation here is to show how the Lie theory for multiplicative graphs is related to the Lie algebras in $\mathcal{L} \mathcal{M}$; for these purposes it is sufficient to consider a narrower class of graphs.

Definition 6.1. A group-like graph $(G \leftleftarrows A)$ over a Lie group $G$ is called a linear Lie graph if the source and target maps are vector bundles over their image, the two-sided action of $G$ on $A$ is linear on the fibres, and the fibres of $s$ and $t$ over $1 \in G$ have the same origin $e \in A$.

Definition 6.2. An augmented rack $\pi: X \rightarrow G$ is called a linear augmented Lie rack if $X$ is a vector space, $G$ is a Lie group, $\pi$ is smooth with $\pi(0)=1$ and the action of $G$ on $X$ is linear.

A linear Lie graph clearly gives rise to a linear augmented Lie rack. The converse is also true. Indeed, the source map $G \times X \rightarrow G$ is simply the projection onto the first factor. The target map sends $(g, x)$ to $g \pi(x)$; its fibre over $h \in G$ is the subspace

$$
\left\{\left(h \pi(x)^{-1}, x\right) \mid x \in X\right\} .
$$

If the point $x \in X$ is taken to be the parameter for the fibre, the left action of $G$ is trivial and the right action is the rack action; both are linear.

An example of a linear Lie graph was given in Section 4.1: the arrows of this graph are directed segments of geodesics on a Lie group, parametrised by length. The corresponding augmented rack is the exponential map of the Lie algebra to the Lie group.

In a linear augmented Lie rack, the map $\pi: X \rightarrow G$ induces a $G$-equivariant ${ }^{9}$ map

\footnotetext{
${ }^{8}$ This should be compared with the definition of a Lie groupoid.

${ }^{9}$ Here, in order to be consistent with the choice of the definition for the Lie algebra in $\mathcal{L} \mathcal{M}$ one has to consider the right action of $G$; accordingly, the adjoint representation should be the right adjoint representation.
} 
of the tangent spaces

$$
\pi_{*}: X=T_{e} X \rightarrow T_{1} G=\mathfrak{g} .
$$

Considering the infinitesimal part of the $G$-action, we see that $\pi_{*}$ is a map of $\mathfrak{g}$ modules and, hence defines a Lie algebra in $\mathcal{L} \mathcal{M}$. Conversely, a Lie algebra in $\mathcal{L} \mathcal{M}$, that is, a homomorphism $\mathfrak{g} \rightarrow \mathfrak{g l}(X)$ which covers the adjoint representation of $\mathfrak{g}$ by means of a map

$$
f: X \rightarrow \mathfrak{g}
$$

for finite-dimensional $\mathfrak{g}$ can be integrated so as to produce a morphism $F$ from a $G$-action on $X$ to the adjoint representation of $G$. Then, the composition

$$
X \stackrel{F}{\rightarrow} \mathfrak{g} \stackrel{\exp }{\longrightarrow} G
$$

defines a linear augmented Lie rack. In terms of racks without augmentation, this exact construction can be found in [9].

Therefore, a Lie algebra in $\mathcal{L} \mathcal{M}$ produces a linear Lie graph. This graph can be thought of as the global integration of the Lie algebra; in this picture, the formal integration (as described in [14]) may be thought of as standing halfway between a Lie algebra in $\mathcal{L} \mathcal{M}$ and the corresponding linear Lie graph.

An augmented rack similar to a linear augmented Lie rack arises from the completion of the graded coinvariant module of an augmented rack. The map of $\mathcal{D}(G)$ modules $\pi_{*}: \mathcal{P}(X) \rightarrow \mathcal{D}(G)$ described in Section 5.5 is a degree 1 map of graded vector spaces and can be extended to the map between the graded completions $\overline{\mathcal{P}(X)}$ and $\overline{\mathcal{D}(G)}$. The image of $\overline{\mathcal{P}(X)}$ lies in the subspace of primitive elements of $\overline{\mathcal{D}(G)}$ and, therefore, the image of the composition

$$
\overline{\mathcal{P}(X)} \stackrel{\overline{\pi_{*}}}{\longrightarrow} \operatorname{Prim} \overline{\mathcal{D}(G)} \stackrel{\exp }{\longrightarrow} \overline{\mathcal{D}(G)}
$$

lies in the group $\mathcal{G}_{0}(\overline{\mathcal{D}(G)})$ of the group-like elements of the complete Hopf algebra $\overline{\mathcal{D}(G)}$. In particular, if $\mathbf{k}=\mathbb{R}$, the rack $\overline{\mathcal{P}(X)} \rightarrow \mathcal{G}_{0}(\overline{\mathcal{D}(G)})$ is a linear augmented Lie rack.

Remark 6.3. There is a situation where a Lie algebra in $\mathcal{L} \mathcal{M}$ can be integrated to a "more non-linear" graph than a linear Lie graph. As mentioned in Section 5.1, differential crossed modules (or, which is the same, crossed modules of Lie algebras) are Lie algebras in $\mathcal{L} \mathcal{M}$. A differential crossed module can be integrated to a crossed module of Lie groups (see [1]), which is an augmented rack that, in turn, gives rise to a group-like graph. The corresponding linear Lie graph can be recovered by taking the tangent spaces to the fibres of the source map of this graph.

\section{Note added in proof}

When the present paper was in press, the author became aware of the paper "The algebra of rack and quandle cohomology" by F. Clauwens (J. Knot Theory Ramifications 20, No. 11 (2011) 1487-1535) which contains the constructions of Section 3.

\section{References}

[1] J. Baez, A. Lauda, Higher-dimensional algebra. V. 2-groups, Theory Appl. Categ. 12 (2004), 423-491. 
[2] A. Crans, Lie 2-algebras, arXiv:math/0409602 [math.QA].

[3] A. Crans, F. Wagemann, Crossed modules of racks, Homology Homotopy Appl. 16 (2014), 85-106.

[4] S. Chmutov, S. Duzhin, J. Mostovoy, Introduction to Vassiliev Knot Invariants, Cambridge University Press, Cambridge, 2012.

[5] R. Fenn, C. Rourke, Racks and Links in codimension two, J. Knot Theory Ramifications 1 (1992), no. 4, 343-406.

[6] R. Fenn, C. Rourke, B. Sanderson, The rack space, Trans. Amer. Math. Soc. 359 (2007), no. 2, 701-740.

[7] M. Forrester-Barker, Group objects and internal categories, arXiv:math/ 0212065 [math.CT].

[8] I.M. James, Reduced product spaces, Ann. of Math. (2) 62 (1955), 170-197.

[9] M. Kinyon, Leibniz algebras, Lie racks and digroups, J. Lie Theory 17 (2007), 99-114.

[10] J.-L. Loday, T. Pirashvili, The tensor category of linear maps and Leibniz algebras, Georgian Math. J. 5 (1998), 263-276.

[11] S. Kamada, Quandles with good involutions, their homologies and knot invariants, in Intelligence of Low Dimensional Topology, 2006, vol. 40 of Ser. Knots Everything, pages 101-108. World Sci. Publ., Hackensack, NJ, 2007.

[12] U. Kraehmer, F. Wagemann, Racks, Leibniz algebras and Yetter-Drinfel'd modules, Georgian Math. J. 22 (2015), 529-542.

[13] S. Matveev, M. Polyak, Cubic complexes and finite type invariants, in Invariants of Knots and 3-Manifolds (Kyoto, 2001), vol. 4 of Geom. Topol. Monogr., pages 215-233. Geom. Topol. Publ., Coventry, 2002.

[14] J. Mostovoy, A comment on the integration of Leibniz algebras, Comm. Algebra 41 (2013), no. 1, 185-194.

Jacob Mostovoy jacob@math.cinvestav.mx

Departamento de Matemáticas, CINvESTAV, Av. IPN 2508, Col. San Pedro Zacatenco, Ciudad de México, C.P. 07360, Mexico 